\title{
ON THIN OR SLENDER BODIES
}

\author{
E. O. TUCK ${ }^{1}$ and Y. M. STOKES ${ }^{\otimes 1}$
}

(Received 10 April, 2012; revised 15 May, 2012)

\begin{abstract}
This is a review of thin-body and slender-body theories, with indications of some new applications. Topics discussed include bodies with near-constant surface pressure, subsonic and supersonic aerodynamics, ship hydrodynamics, slender bodies in Stokes flow, slender footings in elastic media, and slender moonpools. Mathematical features of the thin- and slender-body approximations are also discussed, especially nonlocal convolution terms modelling three-dimensionality in the otherwise two-dimensional near field, end effects, and the role of the logarithm of the slenderness ratio. This review was presented by the first author as the IMA Lighthill Memorial Lecture at the British Applied Mathematics Colloquium (BAMC) 2004.
\end{abstract}

2010 Mathematics subject classification: 76-02.

Keywords and phrases: slender-body theory, fluid mechanics, potential flow.

\section{Introduction}

This paper mainly concerns fluids streaming past impermeable elongated bodies. This topic has always been one of the most important in fluid mechanics, and one to which Sir James Lighthill contributed substantially, from his earliest research days in the 1940s. However, there are also potential or actual applications in other areas, including solid mechanics.

Within fluid mechanics, most of this paper concerns irrotational potential flow of an inviscid incompressible fluid, but again there are many other fluid-mechanical applications, for example, to subsonic and supersonic compressible flows, and to low Reynolds number viscous flows. In all cases, the reference frame adopted is moving with the body and, in this reference frame, the flow is steady and streaming from left to right with unit velocity. The potential flow demands solution of an exterior boundaryvalue problem for Laplace's equation, with a Neumann boundary condition on the body surface.

This is a contribution to the series of invited papers by past ANZIAM medallists (Editorial, issue 52(1)). Ernie Tuck was awarded the 1999 ANZIAM medal and died in 2009.

${ }^{1}$ School of Mathematical Sciences, The University of Adelaide, South Australia 5005, Australia; e-mail: yvonne.stokes@adelaide.edu.au.

(c) Australian Mathematical Society 2012, Serial-fee code 1446-1811/2012 \$16.00 
Even when there is just one such boundary surface, solution of this type of boundary-value problem for geometrically complex bodies presented major computational difficulties until relatively late in the twentieth century, and hence there was often a resort to approximations based on simplifying geometrical features of the body. Fortunately, in many real applications there are indeed such features present, namely the body is elongated in the flow direction, or "streamlined". The now-common English word "streamlined" has a technical origin involving the body geometry conforming to flow streamlines, and this tells us that there is no element of luck about the presence of geometrical simplifying features! Streamlined bodies are usually either thin (approximated by a plane) or slender (approximated by a line).

Nowadays, the tools of computational fluid dynamics are such that this type of boundary-value problem is almost trivially solvable, and one might question the need to use such approximations. However, there are often other reasons for their retention. One such reason is that there may be other boundaries: other bodies, or more importantly other boundaries such as free surfaces. The problem of flow past a ship or submarine is one such example, and the nonlinear boundary conditions on a free surface that is not known in advance present serious difficulties to any computational procedure, even with twenty-first century computers.

Just to set that scene, Figure 1 shows some ship waves computed in a few minutes of $2 \mathrm{GHz}$ PC time using a thin-ship approximation [20]. These computations are for a vessel which approximates in size, shape and speed the ship in the photograph in Figure 2. Capturing the full complexity of a ship-wave pattern is a challenge even after the thin-ship approximation has been made; without that approximation, compromises have to be made that may be unacceptable in some applications, for example, to detection.

We shall return to problems involving free surfaces and other generalizations later, but first let us discuss the most straightforward flow situation, which requires solution of a Neumann problem for Laplace's equation exterior to a single body. For definiteness we shall call this the "prototype" problem.

\section{Interior sources}

Among the many ways to solve Neumann boundary-value problems, one of the oldest is to place singularities inside the body, whose strength is to be determined. We shall only be concerned with nonlifting bodies here, so these singularities can be assumed to be sources. This concept generalizes the Rankine body, which is generated by one source and one equal sink. Good numerical methods can then be devised both in two and three dimensions for arbitrary bodies, with a careful placement of many sources. The source distribution may be discrete or continuous; let us here assume the latter. In the most general case, it is best that the sources lie not too far inside the body, and, in particular, if they lie on the (inside of the) body surface itself, such methods reduce to "boundary integral equation" or "panel" methods.

However, there is a significant class of smooth and not-too-fat bodies for which this principle of closeness to the body surface can be violated without cost. For most 


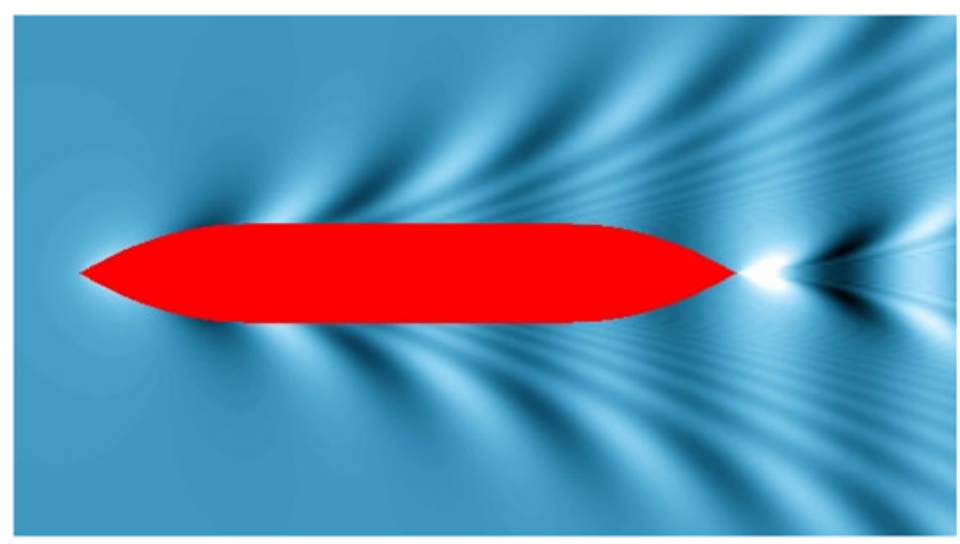

Figure 1. Computed ship waves.

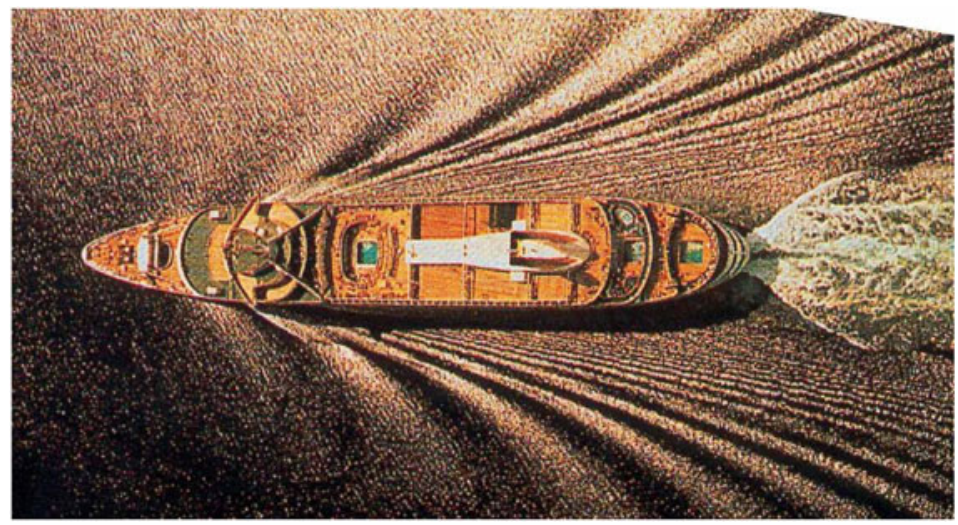

Figure 2. Actual ship waves.

laterally symmetric bodies the sources can be placed on the plane of symmetry, and for most bodies of revolution they can be placed on the axis.

Thus for a body alone in two dimensions (2D) we may suggest

$$
\phi(x, y)=\frac{1}{2 \pi} \int m(\xi) \log \sqrt{(x-\xi)^{2}+y^{2}} d \xi,
$$

and for a body of revolution (with $r$ as a polar coordinate normal to the axis)

$$
\phi(x, r)=-\frac{1}{4 \pi} \int \frac{m(\xi)}{\sqrt{(x-\xi)^{2}+r^{2}}} d \xi .
$$

The integrals in each case take place over a suitable range of $\xi$ values inside the body. For finite bodies, we shall often take this range to be $(-1,1)$, but in principle $(-\infty, \infty)$ 
can be assumed, with truncation to a finite range if $m(\xi)$ vanishes outside that range. Here $\phi$ is the potential for the disturbance to a unit-magnitude $x$-directed stream due to the body, such that the total fluid velocity is $\mathbf{q}=\nabla(x+\phi)$. The only remaining requirement is that the Neumann boundary condition $\mathbf{q} \cdot \mathbf{n}=0$ is satisfied on the body surface, leading to an integral equation for the source-strength function $m(\xi)$.

For example, in the three-dimensional (3D) case on $(-1,1)$, the linearly varying function

$$
m(\xi)=-2 \pi \epsilon^{2} \xi
$$

generates the exact solution for flow over a prolate spheroid which extends somewhat beyond the sources, to $|x|=\zeta$ with $\zeta>1$, and has a maximum radius $r=\sqrt{\zeta^{2}-1}$, where $\epsilon^{2}=-\left[Q_{1}^{\prime}(\zeta)\right]^{-1}$, with

$$
Q_{1}(\zeta)=\frac{1}{2} \zeta \log \frac{\zeta+1}{\zeta-1}-1
$$

a Legendre function of the second kind. We shall further explore this exact solution later. For small $\epsilon$ we have $\zeta \approx 1+\epsilon^{2} / 2$, and the spheroid is slender, with maximum radius/half-length ratio approximately equal to $\epsilon$.

One primitive but often quite successful version of slender-body theory simply says the following. When the body is expanding its cross-section area, each unit-length section occupies an extra volume equal to the rate of increase in section area, and that is volume lost to the stream. To conserve mass, there must therefore be an outward flux or source strength equal to the stream magnitude $U$ times that volume. Hence $m(\xi)$ is approximately equal to $U$ times the $x$-wise rate of change of body section area at station $x=\xi$. Thus, for a body of revolution $r=f(x)$ with section area $\pi f^{2}$ in a unit stream $U=1$,

$$
m(\xi)=2 \pi f(\xi) f^{\prime}(\xi)
$$

In $2 \mathrm{D}$ the section area is replaced by body thickness, so for a symmetric $2 \mathrm{D}$ body $y= \pm f(x)$ with thickness $2 f$ in a unit stream,

$$
m(\xi)=2 f^{\prime}(\xi)
$$

Once $m(\xi)$ is so specified, in principle there is no need for further approximation, and the flow is fully determined. However, let us explore systematic theories in which not only are the source strengths correctly determined by the above formula to a leading order of approximation, but further consistent approximations are made in determining flow quantities of interest such as the pressure distribution on the body.

\section{Thin symmetric bodies in $2 \mathrm{D}$}

A consistent thin-body theory for the symmetric body $y= \pm f(x)$ demands that we consider the limit as $f(x) \rightarrow 0$, in which the body shrinks to the plane $y=0$. The limiting form of the Neumann boundary condition is then

$$
\phi_{y}\left(x, 0_{ \pm}\right)= \pm f^{\prime}(x)
$$


on the top and bottom sides of the limiting plane boundary $y=0$. This boundary condition is satisfied exactly by the expression (2.1), providing $m(\xi)$ is given by (2.5).

The main output is then the pressure distribution (excess over atmospheric) on the body, which is given (neglecting second-order terms) by Bernoulli's equation as

$$
p(x)=-\phi_{x}\left(x, 0_{ \pm}\right) .
$$

Both the fluid density and the stream velocity have been normalized to unity; the nondimensional quantity $p(x)$ used here is then half of the usual pressure coefficient. Note that not only have we neglected the square of the lateral velocity $\phi_{y}$, but also we have consistently evaluated the pressure on the limiting plane boundary $y=0_{ \pm}$rather than the actual boundary $y= \pm f$. Then, using (2.1) and (2.5), we find that the pressure is the Hilbert transform of the body slope, namely

$$
p(x)=-\frac{1}{\pi} \int \frac{f^{\prime}(\xi)}{x-\xi} d \xi .
$$

This is a "small" pressure disturbance, of the same (first-order) magnitude as the body thickness $f$. The relationship (3.1) between shape $f$ and pressure $p$ is linear, and if $f=O(\epsilon)$ for some small parameter $\epsilon$, then also $p=O(\epsilon)$, with error $O\left(\epsilon^{2}\right)$.

For example, on $(-1,1)$ the elliptic cylinder

$$
f(x)=\epsilon \sqrt{1-x^{2}}
$$

gives, according to (3.1), the constant negative pressure $p=-\epsilon$. This is clearly not a good approximation near the ends of the body (where we should find stagnation pressure $p=+1 / 2$ ), a matter to which we shall return later.

There are many properties of Hilbert transforms that can be used to generate interesting results for pressures on thin bodies. For example, since an analytical inverse Hilbert transform is known, we can immediately write down the body slope generating a given pressure distribution. On the full infinite range,

$$
f^{\prime}(x)=\frac{1}{\pi} \int_{-\infty}^{\infty} \frac{p(\xi)}{x-\xi} d \xi,
$$

and on the finite interval $(-1,1)$,

$$
f^{\prime}(x)=\frac{1}{\pi \sqrt{1-x^{2}}} \int_{-1}^{1} \frac{\sqrt{1-\xi^{2}} p(\xi)}{x-\xi} d \xi
$$

(assuming $f(x)=0$ for all $|x| \geq 1$ ).

\section{Slender bodies of revolution}

Not everything follows as easily for slender bodies as it does for thin bodies! Essentially this is because the limiting line is a one-dimensional object, and therefore 
cannot serve as a boundary for the limiting 3D boundary-value problem. Instead of letting the body shrink fully down to the limiting line $r=0$, we must instead match with an "inner" flow for small but nonzero $r$.

We therefore need the behaviour of the potential (2.2) for small $r$, namely

$$
\phi(x, r)=\frac{m(x)}{2 \pi} \log r+b(x)+O\left(m r^{2} \log r\right),
$$

where

$$
b(x)=-\frac{1}{4 \pi} \int m^{\prime}(\xi) \operatorname{sgn}(x-\xi) \log 2|x-\xi| d \xi .
$$

The logarithmic part of (4.1) is easy to understand: a line of 3D sources behaves near that line as if it was a 2D line source with the local source strength. However, if the source strength $m(x)$ varies with $x$, then there will be nonzero and nontrivial axial ( $x$-wise) fluid motion which is determined by the nonlocal term $b(x)$. Equation (4.2) applies both to finite and infinitely long bodies. If the body is of finite length, for example, occupying $|x|<1$, a careful integration by parts yields the alternative formula

$$
b(x)=-\frac{m(x)}{2 \pi} \log 2 \sqrt{1-x^{2}}+\frac{1}{4 \pi} \int_{-1}^{1} \frac{m(x)-m(\xi)}{|x-\xi|} d \xi .
$$

Again, substitution of (4.1) in the approximate Neumann boundary condition $\phi_{r}=f^{\prime}(x)$ on $r=f(x)$ verifies the slender-body approximation (2.4) for the source strength $m(x)$.

Note that $m=O\left(\epsilon^{2}\right)$ if $f=O(\epsilon)$, that is, if $\epsilon$ measures slenderness, and hence the full disturbance potential given by (2.2) is also of second order, with $\phi=O\left(\epsilon^{2}\right)$. The inner approximation (4.1) to $\phi$ with $r=O(\epsilon)$ is strictly then of order $\epsilon^{2} \log \epsilon$. This type of extra " $\log \epsilon$ " factor is common in slender-body theory; for some order-ofmagnitude estimation purposes it can be ignored, and we then view the disturbance due to the body as simply "second order", even near the body. Similarly, it can be shown that the error in the slender-body approximation (2.4) to the source distribution is of order $\epsilon^{4} \log \epsilon$ or (loosely) "fourth order", and the error in the formula (4.1) for the disturbance potential near the body is then of order $\epsilon^{4} \log ^{2} \epsilon$, again loosely fourth order. We shall return to matters involving these logarithms later.

Now the Bernoulli equation determines the pressure as

$$
p=-\phi_{x}-\frac{1}{2} \phi_{r}^{2},
$$

where we have neglected the (fourth order) term $\phi_{x}^{2}$, but now (in contrast to the $2 \mathrm{D}$ case) cannot neglect the (second order) term $\phi_{r}^{2}$. The whole pressure is then of second order. Substituting the inner approximation (4.1) gives, on the body $r=f(x)$,

$$
p(x)=-\left[f(x) f^{\prime}(x)\right]^{\prime} \log f(x)-b^{\prime}(x)-\frac{1}{2} f^{\prime}(x)^{2} .
$$

For finite-length bodies on $(-1,1)$ it is convenient to use (4.3) together with the definitions $A(x)=f(x) f^{\prime}(x)$ and

$$
B(x)=\frac{1}{2} \int_{-1}^{1} \frac{A(x)-A(\xi)}{|x-\xi|} d \xi
$$


to write the pressure as

$$
p(x)=-A^{\prime}(x) \log \frac{f(x)}{2 \sqrt{1-x^{2}}}-B^{\prime}(x)-\frac{x A(x)}{1-x^{2}}-\frac{1}{2} f^{\prime}(x)^{2} .
$$

This expression is in some ways equivalent to the Hilbert transform (3.1) for the corresponding thin-body pressure, and indeed the (linear) integral transform (4.4) connecting $A(x)$ and $B(x)$ plays a similar nonlocal role. However, now there are significant local terms as well, and the full relationship (4.5) between body geometry and pressure distribution is not linear.

The approximation (4.5) clearly breaks down at the ends $x= \pm 1$, where it predicts a (positive) infinite pressure. For most finite bodies, the ends will be stagnation points where we expect $p=1 / 2$. It should of course be noted that this is not as dramatic a failure as appears at first sight. After all, what (4.5) is saying is that the pressure on a slender body is small, of second order in $\epsilon$. On such a scale, the finite stagnation pressure appears effectively infinite.

However, both in $2 \mathrm{D}$ and $3 \mathrm{D}$ it is also possible to construct uniformly valid approximations, and Lighthill [6] was one of the first to do this in 2D. For slender bodies of revolution $r=f(x)$ this simply involves dividing the pressure given by (4.5) by

$$
1+\left(\frac{x f(x)}{1-x^{2}}\right)^{2}
$$

which is such as to guarantee stagnation pressure $p=1 / 2$ at $x= \pm 1$, but makes a negligible $O\left(\epsilon^{4} \log \epsilon\right)$ change if $|x|$ is not near 1 .

\section{Exact pressure on ellipses and spheroids}

If either the elliptic cylinder

$$
y= \pm \epsilon \sqrt{1-x^{2}}
$$

or the spheroid

$$
r=\epsilon \sqrt{1-x^{2}}
$$

is placed in a unit-magnitude stream, the velocity magnitude on its surface is given (exactly) by

$$
q=\frac{1+\lambda}{\sqrt{1+\epsilon^{2} x^{2} /\left(1-x^{2}\right)}}
$$

where $1+\lambda$ is the (maximum) velocity, namely that at $x=0$. This result follows from the exact solutions [4], or simply by careful tangential differentiation of the velocity potential $\phi$, which is (exactly) equal to $(1+\lambda) x$ on these bodies [22]. The corresponding (nondimensional) pressure disturbance $p$ is (exactly)

$$
p(x)=\frac{1}{2}-\frac{1}{2} q^{2}=\frac{-\lambda-\frac{1}{2} \lambda^{2}+\frac{1}{2} \epsilon^{2} x^{2} /\left(1-x^{2}\right)}{1+\epsilon^{2} x^{2} /\left(1-x^{2}\right)} .
$$




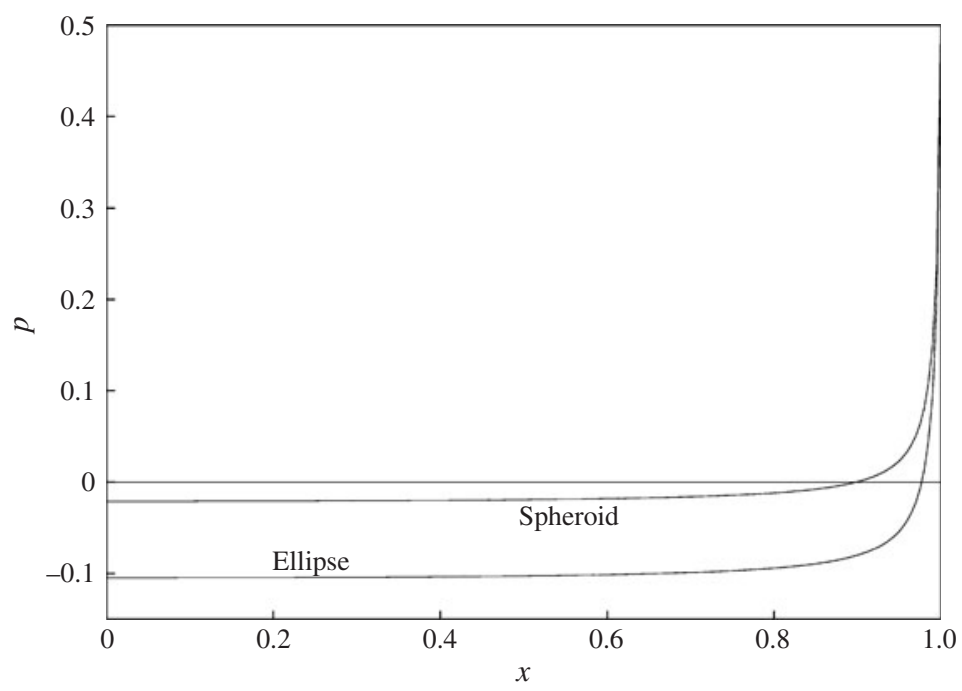

FiguRE 3. Exact nondimensional pressure $p(x)$ on ellipse and spheroid, each with thickness/length $\epsilon=0.1$.

Although the same formula (5.1) yields the surface pressure on ellipses in 2D and spheroids in $3 \mathrm{D}$, the maximum velocity $1+\lambda$ takes different values in each case. For ellipses of width/length ratio $\epsilon$ in $2 \mathrm{D}$ flow we have simply

$$
\lambda=\epsilon .
$$

The exact result in 3D for spheroids of diameter/length ratio $\epsilon$ is a little more complicated, namely

$$
\lambda=-\frac{Q_{1}(\zeta)}{\zeta Q_{1}^{\prime}(\zeta)}
$$

where $\zeta=\left(1-\epsilon^{2}\right)^{-1 / 2}$ and $Q_{1}(\zeta)$ is the Legendre function defined in (2.3).

Figure 3 shows example computations of $p(x)$ in $x \geq 0$, for $\epsilon=0.1$. Although these exact pressures eventually return to the (maximum positive) stagnation value $p=1 / 2$ at the extreme end $x=1$, they take small negative values over more than $90 \%$ of the length.

\section{Thin ellipses and slender spheroids}

We are mainly concerned with thin 2D or slender 3D bodies, for which we need a small- $\epsilon$ approximation. Since such bodies increase the stream velocity by only a small amount, $\lambda$ is small when $\epsilon$ is small; in fact it is at least as small as $\epsilon$ itself. Hence even without further information about the dependence of $\lambda$ on $\epsilon$, we can approximate the denominator of (5.1) by unity, to give (in both 2D and 3D)

$$
p(x)=-\lambda-\frac{1}{2} \lambda^{2}+\frac{1}{2} \frac{\epsilon^{2} x^{2}}{1-x^{2}},
$$




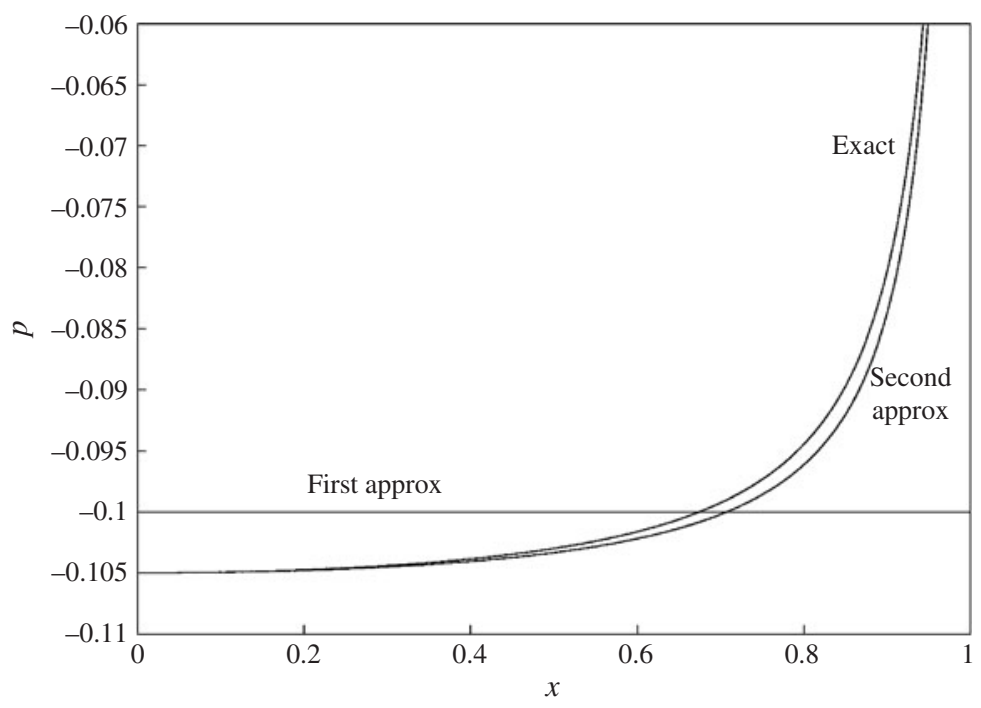

FIGURE 4. Pressure $p(x)$ on ellipse with thickness/length $\epsilon=0.1$.

with error of order at most $\epsilon^{3}$. But then, since $\lambda$ is small, we can also neglect the term in $\lambda^{2}$ relative to that in $\lambda$, giving

$$
p(x)=-\lambda+\frac{1}{2} \frac{\epsilon^{2} x^{2}}{1-x^{2}},
$$

now with errors of order at most the maximum of $\lambda^{2}$ and $\epsilon^{3}$.

This is most obvious in 2D for ellipses, since $\lambda=\epsilon$ exactly and the error in (6.2) is of order $\epsilon^{2}$. But in that case the second term of (6.2) is also small within the above error range, and can and should be neglected relative to the first term (so long as $|x|$ is not too close to unity), leading to the constant pressure

$$
p(x)=-\epsilon
$$

on thin ellipses, a result with order $\epsilon^{2}$ error, as already obtained by the thin-body theory. If we revert to (6.1), we obtain a more accurate but nonconstant result for ellipses, of the form

$$
p(x)=-\epsilon-\frac{1}{2} \epsilon^{2}+\frac{1}{2} \frac{\epsilon^{2} x^{2}}{1-x^{2}},
$$

now with formal error of order $\epsilon^{3}$, but which happens to be exact at $x=0$. Both approximations are displayed (as the horizontal line "first approx" and the curve "second approx", relative to the "exact' solution) in Figure 4 for $\epsilon=0.1$.

Again the 3D result for spheroids is somewhat more complicated. Now by small- $\epsilon$ expansion of the exact result (5.2) we find a much smaller relative increase in velocity, 


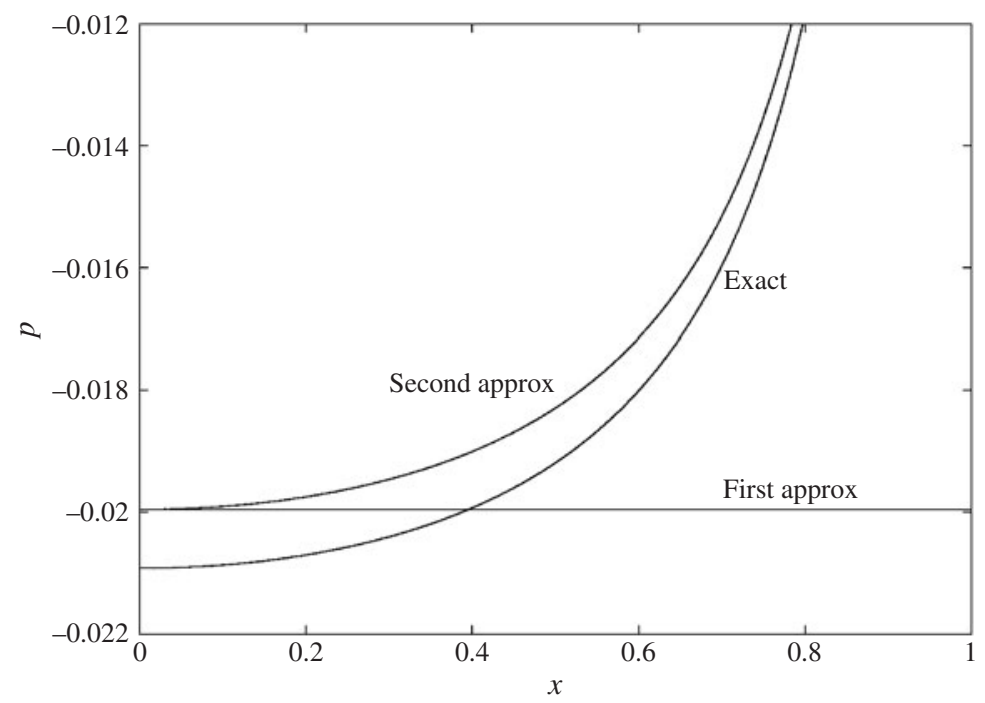

FIGURE 5. Pressure $p(x)$ on spheroid with slenderness $\epsilon=0.1$.

namely

$$
\lambda=-\epsilon^{2}\left[\log \frac{\epsilon}{2}+1\right]+O\left(\epsilon^{4} \log ^{2} \epsilon\right)
$$

So (6.2) becomes

$$
p(x)=\epsilon^{2}\left[\log \frac{\epsilon}{2}+1\right]+\frac{1}{2} \frac{\epsilon^{2} x^{2}}{1-x^{2}},
$$

which has an error of order $\epsilon^{4} \log ^{2} \epsilon$. Equation (6.3) is thus a very accurate approximation so long as $|x|$ is not too close to unity, but does not predict constant pressure on slender spheroids. It is displayed for $\epsilon=0.1$ as the "second approx" curve of Figure 5, relative to the "exact" curve.

The approximation (6.3) also follows from the general slender-body formula (4.5): noting that for the spheroid $f(x)=\epsilon \sqrt{1-x^{2}}$ we have $A(x)=B(x)=-\epsilon^{2} x$ and the argument of the logarithm in (4.5) is $\epsilon / 2$, the first two terms of (4.5) yield the constant part of (6.3) while the remaining two terms yield the nonconstant part. The denominator of (5.1) is also restored if the end correction factor (4.6) is applied.

Is the pressure on a slender spheroid in 3D then not constant to a leading order of approximation, as it was on a thin ellipse in 2D? Well, yes, in a sense the pressure can be said to be constant, but only as a very crude first approximation. If we drop the second term of (6.3), we leave a constant pressure

$$
p(x)=\epsilon^{2}\left[\log \frac{\epsilon}{2}+1\right]
$$


on a slender spheroid, but we have now made an absolute error of order $\epsilon^{2}$. The constant term (6.4) that then remains is of order $\epsilon^{2} \log \epsilon$, so we have made a relative error of order $1 / \log \epsilon$, and $\epsilon$ must be exceedingly small for this to be justified. The approximation (6.4) at $\epsilon=0.1$ is shown as the horizontal line labelled "first approx" in Figure 5.

The danger of neglecting order $\epsilon^{2}$ terms relative to order $\epsilon^{2} \log \epsilon$ terms is illustrated by the fact that if we do wish to drop the second term of (6.3), consistently we should also drop the " +1 " inside the square bracket of the first term and even the factor " $1 / 2$ " inside the logarithm, since these terms also contribute order $\epsilon^{2}$ to the final answer. In fact, with the same formal order $\epsilon^{2}$ error, (6.4) can be replaced by

$$
p(x)=\epsilon^{2}[\log \epsilon+k]
$$

for any finite constant $k$. It is hardly comfortable to have to deal with an approximation which has such a large element of nonuniqueness.

However, one redeeming feature of the approximation (6.4), or of (6.5) with $k=1-\log 2 \approx 0.31$, is that it is a good approximation (with much smaller formal error, of order $\epsilon^{4} \log ^{2} \epsilon$ ) to the pressure at the centre station $x=0$, where the second term of (6.3) vanishes. Hence the "first approx" and "second approx" agree at $x=0$ in Figure 5. This enables a quantitative measure of constancy of pressure. In general, as $x$ varies the pressure coefficient rises from its (small negative) value at $x=0$, and we may ask how small must $\epsilon$ be in order that this rise is small relative to the pressure magnitude at $x=0$.

For example, suppose that we fix attention on a point where $x^{2}=1 / 2$, or $x \approx 0.7$. Then the absolute rise in $p$ between the centre station and this station is $\epsilon^{2} / 2$, both for ellipses and spheroids. However, this is relatively much more significant in the latter case than the former. Thus in order for the pressure magnitude at this point to be at most $10 \%$ different from that at $x=0$, it is only necessary for $\epsilon$ to be smaller than 0.2 for ellipses, but $\epsilon$ must be smaller than $2 e^{-6} \approx 0.005$ for spheroids. This comparison is even more severe closer to the ends; for example, if we require such pressure constancy as far from the centre as $x^{2}=2 / 3$ or $x \approx 0.8$, where the absolute rise is $\epsilon^{2}$, then we require $\epsilon<0.1$ for ellipses, but a quite unrealistic $\epsilon<2 e^{-11} \approx 0.000033$ for spheroids.

\section{Slender bodies with near-constant pressure}

If the pressure is not constant on a spheroid, is there any body of revolution on which the pressure is constant? The exact answer is certainly no, but we only require a slender-body approximation. Specifically, we seek shapes $f(x)$ such that the pressure $p(x)$ given by (4.5) takes a constant (small negative) value $p_{0}$, for all $x$ not close to the ends. This can be done in a straightforward numerical manner by forcing $p\left(x_{n}\right)=p_{0}$ at a set of $N$ collocation points $x=x_{n}, n=1, \ldots, N$, which can lie in $0 \leq x<1$ using symmetry. If we specify $f(x)$ by a set of $N$ parameters, this yields a set of $N$ nonlinear algebraic equations in the $N$ unknown parameters, which can be solved by Newton's method. 


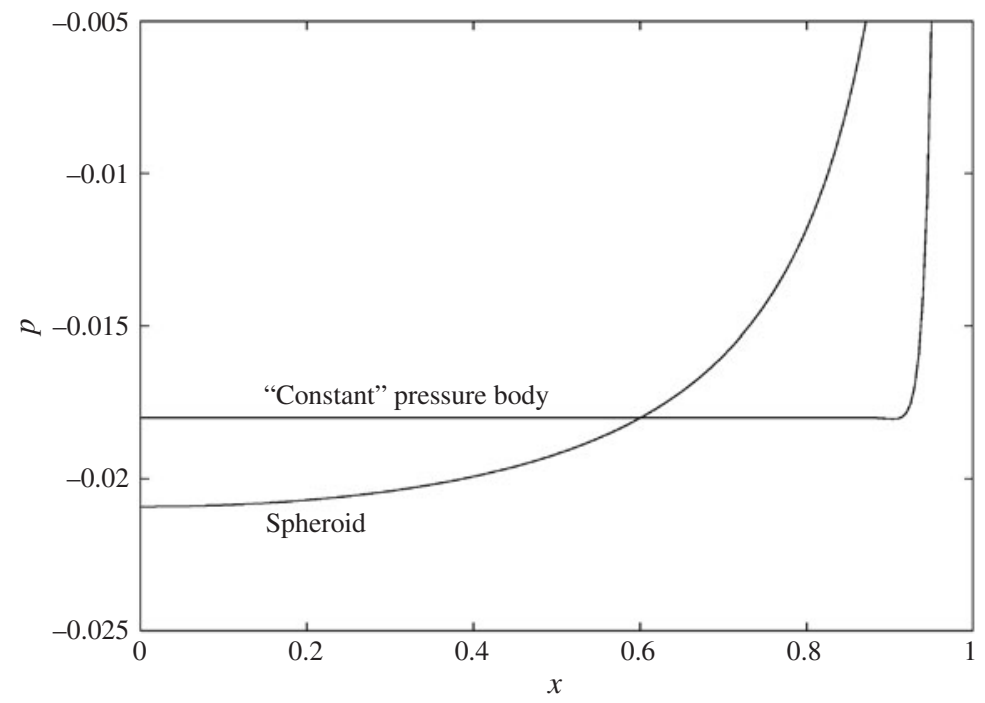

Figure 6. Pressure $p(x)$ on "constant" pressure body with slenderness $\epsilon=0.1$, compared to exact pressure on spheroid with the same slenderness.

A convenient parameterization of the body shape is obtained by the Legendre series

$$
A(x)=\sum_{n=1}^{N} a_{n} P_{2 n-1}(x),
$$

where $P_{2 n-1}(x)$ are the Legendre polynomials and $a_{n}$ are the $N$ coefficients to be determined. Then

$$
B(x)=\sum_{n=1}^{N} a_{n} \sigma_{2 n-1} P_{2 n-1}(x),
$$

where $\sigma_{n}=1+1 / 2+1 / 3+\cdots+1 / n$, and

$$
\frac{1}{2} f(x)^{2}=\int_{1}^{x} A(x) d x=\sum_{n=1}^{N} \frac{a_{n}}{4 n-1}\left[P_{2 n}(x)-P_{2 n-2}(x)\right] .
$$

So all quantities required for (4.5) can be computed readily, given the coefficients $a_{n}$. The spheroid is the particular case $N=1$, with $a_{1}=-\epsilon^{2}$.

The numerical results for large $N$ depend to a certain extent on the choice of the collocation points $x_{n}$, and ultimate refinement of this choice and of the present numerical method was not sought. A satisfactory grid was $x_{n}=(n-1) /(N+1)$, for which convergence (for example, of $f(0)=\epsilon$ as $N$ increases) to about four figures was achieved by $N=22$. The coefficients $a_{n}$ decrease in size quite rapidly, with, for example, $a_{20}$ generally of the order of $10^{-8}$ compared to $a_{1}$ of the order of $10^{-2}$. The final pressure is constant to more than four figures not only at the grid points, but also 


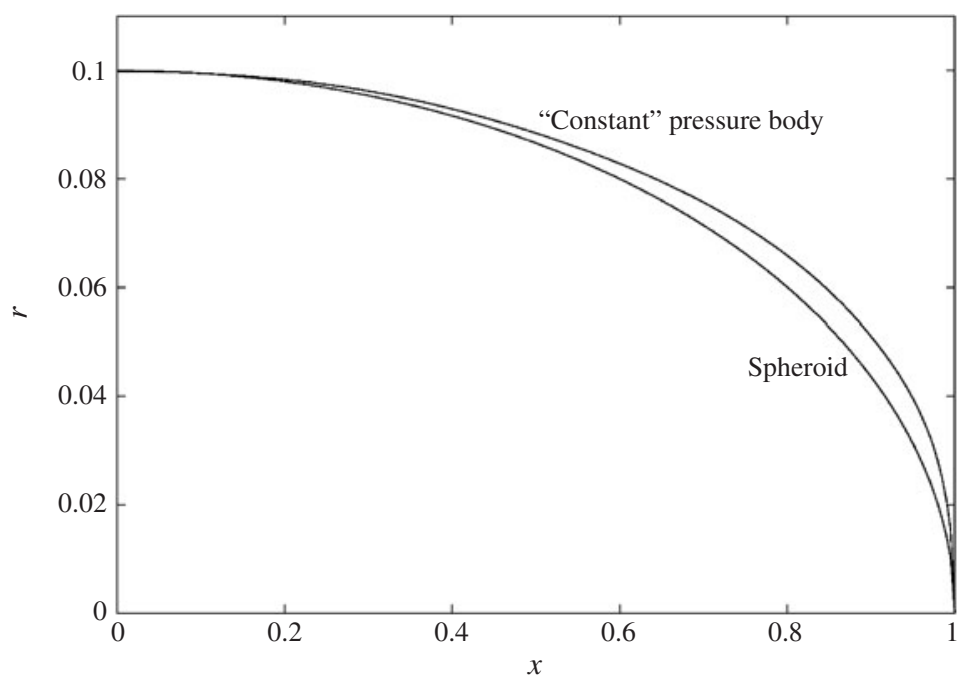

FIGURE 7. Shape $r(x)$ of "constant" pressure body compared to spheroid.

for all $x$ less than $x_{N-1}$. It is then constant to three figures (falling slightly) between $x_{N-1}$ and $x_{N}$, before increasing rapidly for $x>x_{N}$, that is, for $x>0.91$ at $N=22$. Even so, at $N=22$ the pressure remains within $10 \%$ of $p_{0}$ up to $x=0.93$.

It is clearly impossible to maintain constancy of pressure right to the ends. This is true whether or not the uniformizing factor (4.6) is used; with it the extreme end pressure is finite, without it infinite, but in neither case is the end pressure small and negative. In practice, it made very little difference to the numerical results whether (4.6) was or was not used, and loss of pressure constancy in about the last $7 \%$ of the length seems inevitable. Further work is needed to assess whether this breakdown range can be reduced. In any case, the present extent of constancy of pressure far exceeds that for a spheroid, as indicated by the comparison in Figure 6 for $\epsilon=0.1$.

Figure 7 shows the final body shape $r=f(x)$ at $p_{0}=-0.018$, which generates a body with width/length ratio $\epsilon=0.10$, compared to a spheroid of the same slenderness. As we increase $\left|p_{0}\right|$, the body becomes less slender, and eventually the procedure fails. This is signalled first by a loss of convexity at about $p_{0}=-0.030$ or $\epsilon=0.14$, with $f^{\prime}(0)>0$. All "constant" pressure bodies less slender than this are dumbbell-shaped.

Within the whole range of convex bodies, the computed relation between slenderness $\epsilon$ and pressure $p=p_{0}$ is quite accurately represented by (6.5) with $k=0.50$, which is to be compared to $k=0.31$ for the spheroid result (6.4).

\section{Relationship to long cavities}

Although in principle the present work is independent of the particular application to supercavitation, this area is one where there has been a significant amount of 


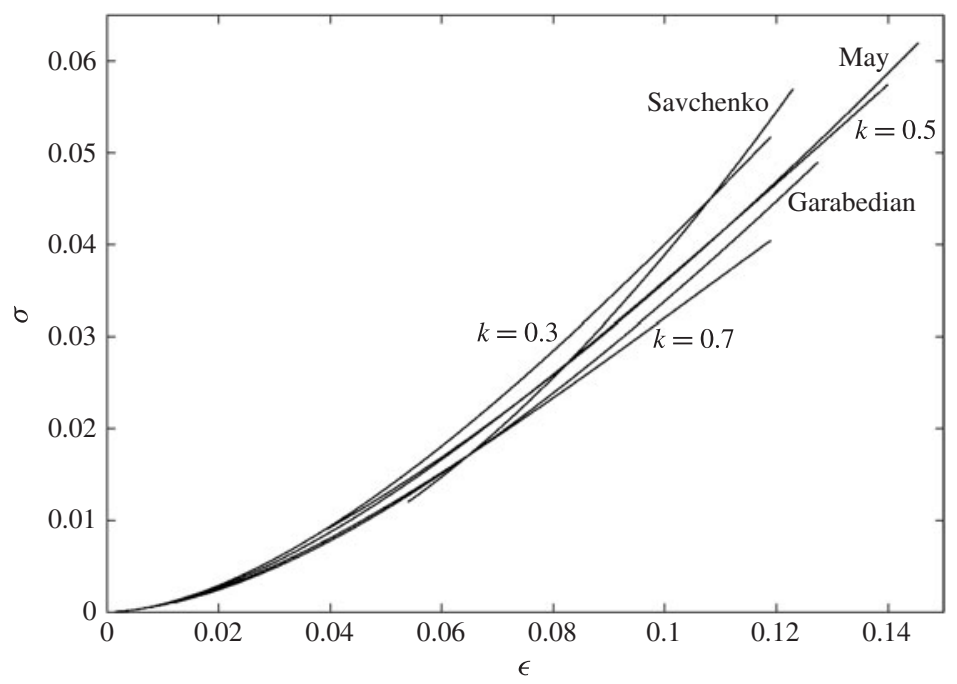

Figure 8. Cavitation number $\sigma$ versus slenderness $\epsilon$ from (8.1) with $k=0.3,0.5,0.7$, compared to supercavitation results of May [8], Savchenko [14] and Garabedian [1].

experimental, empirical and computational effort; see, for example, the paper by Tulin [22] and several review articles edited by Michel [9]. In this application the closed "body" of interest is a combination of a relatively short rigid "cavitator" or nose, which has a prescribed shape and on which the pressure is not necessarily constant, a much longer attached cavity, on which the pressure is required to be constant, and another short region of cavity collapse at the trailing end. In supercavitation the constant negative nondimensional pressure is written as $p_{0}=-\sigma / 2$, where $\sigma$ is the "cavitation number".

Hence the present theory is relevant to the slender-body limit in which the cavity length far exceeds the cavitator length and the cavitation number is small. In a sense this is no different from the numerical consequences in the previous section of seeking approximate solutions for "constant" pressure bodies, where we must allow the pressure to be nonconstant near the ends of the body. Those end regions can then act as surrogates for the cavitator and collapse region, and indeed Reichardt's early study of supercavitation [13] has already used this approach.

Significant interest in supercavitation lies in relationships between cavitator geometry and cavity dimensions. There are empirical formulae indicating that the length and width of the cavity (relative to the length of the cavitator) are each proportional to the square root of a drag coefficient which characterizes the geometry of the cavitator. Hence the slenderness $\epsilon$ or width/length ratio of the cavitatorcavity combination is (at least according to these formulae) independent of the drag coefficient, and thus independent of the shape of the cavitator, depending only on the cavitation number $\sigma$. 
For two-dimensional supercavitation, the thin-body theory of Tulin [21] indicates that $\sigma=2 \epsilon$, and that the cavity is asymptotically elliptical. This agrees with the conclusion that, to leading order in $\epsilon$, the pressure on a thin ellipse is constant and of magnitude $p_{0}=-\epsilon$, with a consistently small $O\left(\epsilon^{2}\right)$ error.

The equivalent result for axisymmetric three-dimensional supercavitation is a little more problematic. If we are prepared to neglect terms with relative error $O(1 / \log \epsilon)$, then the cavity is asymptotically spheroidal and (6.5) implies that

$$
\sigma=-2 \epsilon^{2}(\log \epsilon+k)
$$

for some constant $k$. This constant $k$ is formally arbitrary, although if the cavity were exactly spheroidal, $k=0.31$ might be preferred. However, the cavity is only spheroidal to a very crude approximation, and if we abandon the spheroidal assumption then there is no formal asymptotic justification for (8.1) at any $k$. On the other hand, if the cavity asymptotes to one of the "constant" pressure bodies constructed in the previous section, then since we have found that these bodies have a pressure close to (6.5) with $k=0.50$, a case can be made for use of (8.1) with $k \approx 0.5$.

Figure 8 shows graphs of $\sigma$ versus $\epsilon$ from (8.1) for $k=0.3,0.5,0.7$, compared to supercavitation results of May [8], Savchenko [14] and Garabedian [1]. The May and Savchenko curves are empirical fits to experimental measurements. The May curve is very close to the present $k=0.5$ curve. The Savchenko curve is close to $k=0.7$ at the more slender end of the experimental range, and to $k=0.3$ at the less slender end, with $k=0.5$ being a reasonable compromise value. The Garabedian curve is a theoretical formula based on neglect of terms with relative error $O(1 / \log \sigma)$, which is almost (but not quite!) the same thing as neglect of terms with relative error $O(1 / \log \epsilon)$, and seems to give results between $k=0.6$ and $k=0.7$, which are also very close to an empirical formula of Reichardt [13]. Other experimental, empirical or computational results are quoted in articles edited by Michel [9], and also fall in the approximate range $0.3<k<0.7$ of Figure 8 .

\section{Other applications of slender-body theory}

9.1. General inner limit and drag Let us turn to more general slender-body theories. There are a number of such theories which use a distribution of 3D or point sources along the $x$-axis, with a varying strength $m(x)$ which often can be shown to be equal to $U S^{\prime}(x)$, where $U$ is the free stream velocity (normalized here to unity) and $S(x)$ is the body section area. If the potential of a unit 3D source at the origin is written as $G_{3}(x, y, z)$, we need the limit of such a line distribution as $r=\sqrt{y^{2}+z^{2}} \rightarrow 0$, and expect this to be of the form

$$
\int m(\xi) G_{3}(x-\xi, y, z) d \xi \longrightarrow m(x) G_{2}(y, z)+b(x),
$$

where

$$
b(x)=\int m^{\prime}(\xi) F(x-\xi) d \xi
$$


Here $G_{2}(y, z)$ is the corresponding unit 2D or line source potential in the $(y, z)$ crosssection plane, and $F(x)$ is a suitable kernel function. At each fixed station $x$, the first term on the right of (9.1) is "local", depending only on the current value of $m(x)$. If the source strength $m(x)$ is not constant, there is an additional "nonlocal" term $b(x)$, whose value at station $x$ in principle depends on values of the axial rate of change of source strength $m^{\prime}(\xi)$ at all other stations $\xi$, expressed as a convolution integral (9.2) with weighting function $F(x-\xi)$.

For the simple prototype problem considered so far, namely irrotational flow of an incompressible inviscid fluid past a single fixed impermeable body,

$$
\begin{aligned}
G_{3}(x, y, z) & =-\frac{1}{4 \pi} \frac{1}{\sqrt{x^{2}+r^{2}}}, \\
G_{2}(y, z) & =\frac{1}{2 \pi} \log (r)
\end{aligned}
$$

and

$$
F(x)=-\frac{1}{4 \pi} \operatorname{sgn}(x) \log 2|x| .
$$

Here the nonlocal term captures the essential elliptic character of the prototype boundary-value problem; what we do at one point of space affects what happens everywhere else.

Generalizations of the prototype problem sometimes involve asymptotic results similar to (9.1) with different 3D source potentials $G_{3}$ on the left, but the same or similar functions $G_{2}$ and $F$ on the right. In particular, there are a number of slenderbody problems in which the $2 \mathrm{D}$ source $G_{2}$ is still given by (9.4) even though $G_{3}$ and $F$ are different. Physically, this can be interpreted as saying that although the $3 \mathrm{D}$ problem differs from the prototype, that difference disappears in the 2D near field in the crosssection plane. However, axial changes in the parameters of that (incompressible) 2D flow induce axial velocities via $b(x)$ which are nonlocal and capture the unique threedimensionality of the problem.

Various important output quantities follow from the above formalism. For example, local terms (both that already in (9.1) and others arising from its use to generate the pressure) do not contribute to the drag on the body, which is then due to a contribution $-b^{\prime}(x)$ to the pressure depending on $x$ alone. Hence the drag is $D=\int S^{\prime}(x)\left(-b^{\prime}(x)\right) d x$, where $S(x)$ is the cross-section area. So after integration by parts,

$$
D=\int d x S^{\prime \prime}(x) \int d \xi S^{\prime \prime}(\xi) F(x-\xi) \text {. }
$$

Hence d'Alembert's paradox $D=0$ is verified for the prototype problem, and indeed for any generalization where $F(x)$ remains an odd function of $x$ as in (9.5). Otherwise, in evaluating the drag by (9.6) we can replace $F(x)$ by its even part.

9.2. Compressibility Inclusion of compressibility of the fluid provides a direct generalization of the prototype problem. Then at free-stream Mach number $M$, the 
3D source function is simply obtained by replacing $r^{2}$ with $\left(1-M^{2}\right) r^{2}$ in (9.3) for the prototype (incompressible) problem. For subsonic flow with $M<1$ this can always be done, and the net effect is essentially as if $F(x)$ was still given by (9.5). The boundaryvalue problem remains elliptic, and d'Alembert's paradox $D=0$ still holds.

For supersonic flow with $M>1$, the 3D source potential must vanish outside of the Mach cone $r=x / \beta$, where $\beta=\sqrt{M^{2}-1}$, so the range of integration is truncated to $\xi<x-\beta r$. Then we find as we let $r \rightarrow 0$ that the local term is again given by the incompressible 2D line source (9.4), while the nonlocal term has kernel

$$
F(x)=-\frac{1}{2 \pi} \log \frac{2}{\beta} x
$$

if $x>0$, and zero otherwise. Now although $b(x)$ still describes nonlocal effects, it only carries a downstream influence, the contribution at station $x$ only depending on $m(\xi)$ for $\xi \leq x$, thus capturing the essential hyperbolic nature of the original 3D boundaryvalue problem. Now $F(x)$ is no longer an odd function, and the drag is

$$
D=-\frac{1}{4 \pi} \int d x S^{\prime \prime}(x) \int d \xi S^{\prime \prime}(\xi) \log |x-\xi| .
$$

This supersonic result was obtained by von Kármán and Moore [3], and improved upon by Lighthill [5]. In fact, the supersonic slender-body theory was obtained before corresponding incompressible or subsonic slender body theories were derived [24].

9.3. Slender ships Another qualitatively straightforward generalization is to slender ships moving over a free surface, where the linearized disturbance potential must satisfy, on the plane $z=0$, the Kelvin boundary condition

$$
g \phi_{z}+\phi_{x x}=0
$$

where $g$ is gravity. Then the unit point source potential $G_{3}$ satisfying (9.7) is given by a very complicated formula [25, p. 484], involving a double integral with respect to the wave number and direction of the water waves. However, again, the local term in (9.1) is just given by the simple line source (9.4). This now reflects the fact that the near-field limit of the Kelvin condition is $\phi_{z}=0$, so the plane $z=0$ is a plane of symmetry not supporting waves. This has an immediate local consequence, in that the apparent source strength is related to the section area of the double body, the ship section being reflected in $z=0$. Thus if $S(x)$ now denotes the true submerged ship section area, $m(x)=2 S^{\prime}(x)$.

However, the wavelike nature of the original 3D flow is retained in the near field by the nonlocal term $b(x)$, which now has the kernel function

$$
F(x)=-\frac{1}{4 \pi} \operatorname{sgn}(x) \log 2|x|-\frac{1}{8}\left[\mathbf{H}_{0}(g x)+(2+\operatorname{sgn}(x)) Y_{0}(g|x|)\right],
$$

where $\mathbf{H}_{0}$ is a Struve function and $Y_{0}$ a Bessel function. The first term of (9.8) is given by (9.5) as in the prototype problem, and the remaining terms are wavelike. If 
we formally let $g \rightarrow \infty$, these wavelike terms disappear, and in that limit the Kelvin condition becomes (everywhere) the symmetry condition $\phi_{z}=0$, so the problem has reduced to the prototype problem for the double body.

Since $\mathbf{H}_{0}$ is odd, the even part of the kernel $F(x)$ is $-\frac{1}{4} Y_{0}(g|x|)$, and the drag (9.6) is

$$
D=-\frac{1}{2} \int d x S^{\prime \prime}(x) \int d \xi S^{\prime \prime}(\xi) Y_{0}(g|x-\xi|),
$$

which is the slender-ship wave-resistance formula obtained independently by Vossers [23], Maruo [7] and Tuck [15].

In fact, Maruo obtained (9.9) simply by approximating Michell's thin-ship waveresistance integral for small draft [10]. That is, in ship hydrodynamics, to a leading order of approximation, slender-body theory is subsumed in thin-body theory. It is interesting that Michell's integral demands information about the complete offsets $y= \pm Y(x, z)$ of the ship, whereas the slender-ship formula (9.9) demands only a knowledge of the ship's section area $S(x)=2 \int Y(x, z) d z$. If a computer program for Michell's integral is available, there is no need for a separate computer program for the slender-ship integral (9.9), since one can simply run the thin-ship code for artificial rectangular sections with offsets $Y(x, z)=S(x) /(2 d)$ and an arbitrary small draft $d$.

However, this subsumption does not apply at higher orders of approximation. Thus, as with the prototype problem, the leading-order slender-ship potential is of second order in the slenderness or beam/length ratio $\epsilon$. In the prototype problem, the next correction to the inner-region potential is of fourth order in $\epsilon$. However, in the presence of a free surface this is no longer the case, and the next term is larger, of third order in $\epsilon$. Not only that, but it depends on nonlinear free-surface terms neglected in the Kelvin boundary condition (9.7). Hence this correction cannot be subsumed in (linear) thin-ship theory. The effect on the drag is that the slender-ship formula (9.9) gives a fourth-order result, which is subject to a nonlinear fifth-order correction. It would be of considerable interest to compute this correction to the wave resistance, but this does not seem to have been done.

9.4. Stokes flows past slender bodies We now turn to applications of slender-body theory where the source strength $m(x)$ is no longer given by the axial rate of change of body section area. One such application is to viscous flow, described by the Stokes equations [16]. Then internal (irrotational) sources alone are not sufficient to represent the flow about a fixed body, and we also need point singularities generating rotational flow, and contributing to drag forces. For slender bodies of revolution $r=f(x)$, the appropriate generators are then axial distributions of both point sources and point "Stokeslets".

It turns out that, in this problem, the source distribution plays a minor role, not contributing to the leading-order slender-body approximation. If the Stokeslet strength is now written as $m(x)$, satisfaction of the no-slip boundary condition on $r=f(x)$ demands that [16]

$$
\frac{m(x)}{2 \pi}\left[\log f(x)+\frac{1}{2}\right]+b(x)=-\frac{1}{2},
$$


or on $(-1,1)$ that $m(x)$ satisfy the integral equation

$$
\frac{m(x)}{2 \pi}\left[\log \frac{f(x)}{2 \sqrt{1-x^{2}}}+\frac{1}{2}\right]+\frac{1}{4 \pi} \int_{-1}^{1} \frac{m(x)-m(\xi)}{|x-\xi|} d \xi=-\frac{1}{2} .
$$

Although the Legendre series (6.1) provides a convenient way to discretize (9.10) for numerical purposes, there are a number of serious difficulties, especially at the ends, of a character similar to that encountered earlier when attempting to force the pressure in the prototype problem to be everywhere constant. A simple alternative [17] is to use inverse methods, in which $m(x)$ is given and the shape $f(x)$ is determined by (9.10).

9.5. Solid mechanics for elongated loads A somewhat similar application in solid mechanics $[2,18]$ is to a "slender punch", that is, a load applied to a finite elongated region of the plane free boundary of an elastic half-space. This has applications to elongated footings for buildings. The similarity to Stokes flow arises from the fourthorder nature of the partial differential equations describing the problem.

Suppose that the punch lies in $|y|<f(x)$, and let $m(x)$ be the (downward) force per unit length that it exerts. Then the downward displacement $w(x)$ under the punch can be shown to be proportional to the potential $\phi$ of a line of sources $m(x)$ as in the prototype problem, that is, as given in the near field by (4.1), evaluated for frictionless punches at $r=f(x) / 2$. Specifically,

$$
\frac{1}{2} \frac{\mu}{1-v} w(x)=\frac{m(x)}{2 \pi} \log \left[\frac{1}{2} f(x)\right]+b(x),
$$

where $\mu$ and $v$ are the elastic (Lamé) constants. If there is adhesion, the point of evaluation differs from $r=f(x) / 2$, but one can then define an "effective frictionless width" [18] replacing and increasing the actual punch width $2 f(x)$.

If the load $m(x)$ is given, on a given punch width $2 f(x)$, equation (9.11) with $b(x)$ determined from $m(x)$ by (4.2) or (4.3) gives the resulting displacement $w(x)$ directly. However, again it is a much more difficult task to solve for the load $m(x)$ given the displacement $w(x)$, since we then have to solve the integral equation

$$
\frac{1}{2} \frac{\mu}{1-v} w(x)=\frac{m(x)}{2 \pi} \log \left[\frac{f(x)}{4 \sqrt{1-x^{2}}}\right]+\frac{1}{4 \pi} \int_{-1}^{1} \frac{m(x)-m(\xi)}{|x-\xi|} d \xi
$$

for $m(x)$. Equation (9.12) is quite similar to (9.10) for slender-body Stokes flow, and leads to similar difficulties at the ends $[12,18]$.

9.6. Slender moonpools Let us mention a final application of slender-body ideas, even more remote from the original prototype problem, in that it does not involve a streaming flow, or indeed even a "body". The application is to sloshing of water waves in an elongated basin. If this basin is fully enclosed, as in a swimming pool or lake, there are interesting asymptotic issues for slender basins, concerned with the seiching modes of motion along the basin, but these issues have little in common with the source-distribution models discussed in the present paper. In particular, if the water 


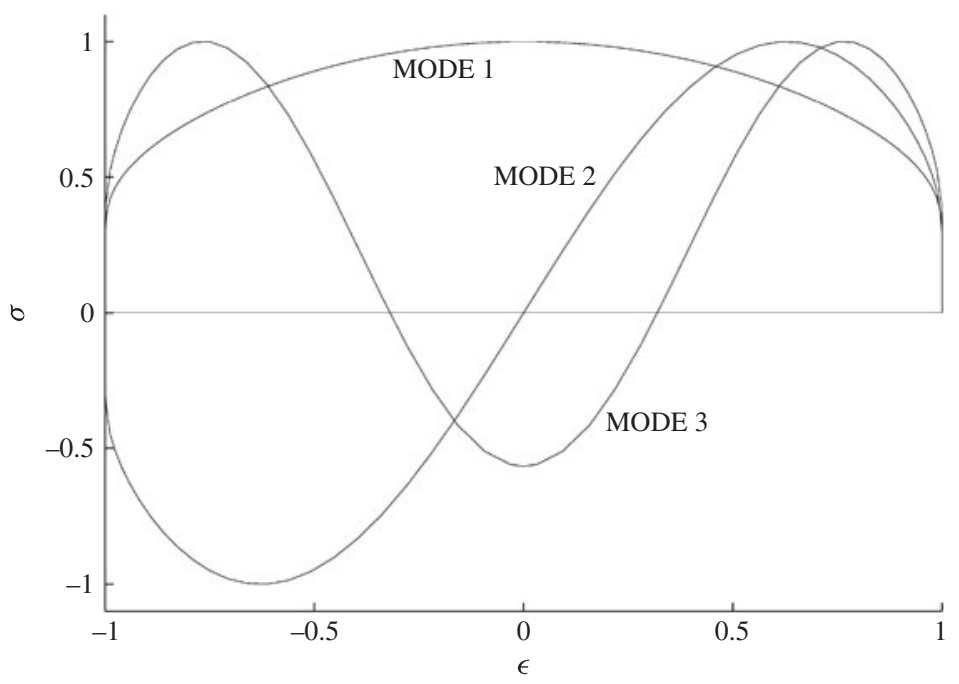

FIGURE 9. The first three longitudinal moonpool modes.

level at one station $x$ falls, it must rise instantly at other stations to conserve mass, and the flow in the bounded cross-section at station $x$ cannot have a source-like character.

However, suppose that the "basin" is not closed, having access at each station $x$ to an ocean of infinite depth. Then when the water level at that station falls, the outward volume flux so generated can pass to infinity in a source-like manner. The water level then need not immediately rise at other stations.

For example, the basin may be a "moonpool" [11], a hole giving access to water beneath an otherwise rigid boundary. This boundary could be the bottom of the hull of a drilling ship, or perhaps an ice sheet. Here we idealize the boundary to be a rigid sheet of infinite extent and zero thickness, and the water to be of infinite extent and depth, and let the moonpool be elongated in the $x$-direction.

Now if sloshing is taking place in this moonpool, with a water level varying with $x$, there will be an apparent source at each station $x$, of some strength $m(x)$ to be determined, and hence if the moonpool is slender, the flow will appear in the far field to be generated by a line distribution of point sources of strength $m(x)$ as in (2.2). Note that there are no waves in the far field, since the "free" surface there is rigid!

On the other hand, if we move into the near field, with $r$ reduced to the size of the width of the moonpool, we shall see water waves, with a rise or fall of the water level in the moonpool. Hence we need again the inner limit (9.1). Details of the 2D wave-like inner flow are given by Tuck and Newman [19].

However, what is clear without such detail is that, for moonpools with a rectangular planform, there is a necessity for the whole disturbance potential $\phi$ in the near field as given by (9.1) to have the identical $x$-variation along the pool. That is, the nonlocal term $b(x)$ needs to be proportional to the local source strength $m(x)$. If the 
proportionality constant is $\mu$, we therefore have to solve an eigenvalue problem, for the integro-differential equation

$$
\int m^{\prime}(\xi) F(x-\xi) d \xi=\mu m(x)
$$

with $F(x)$ given by $(9.5)$. For a pool in $(-1,1)$, the truncated Legendre series $(7.1)$ is a convenient tool for numerical solution of this problem, and the first three eigenvalues in increasing order are given by

$$
2 \pi \mu+\log 2=0.2332,1.4437,1.9409 .
$$

The first three eigenmodes $m(x)$ are shown in Figure 9. The lowest-order mode is a symmetric "pumping" mode which does not have an equivalent for a closed pool, as there is a nonzero net volume of water-surface displacement which would not conserve mass if the pool was closed. The second (antisymmetric) mode has a nodal point at the centre station $x=0$ and is the fundamental longitudinal "sloshing" mode. The third mode is symmetric with two nodal points, and is also of a pumping nature. All of these modes seem numerically to have infinite slope at the ends $|x|=1$, though this matter has not yet been investigated analytically.

\section{Editorial note}

This review was prepared for and presented as the IMA Lighthill Memorial Lecture at the British Applied Mathematics Colloquium (BAMC) 2004. Subsequent to Professor E. O. Tuck's death (11 March 2009), the editors of the ANZIAM Journal decided to invite the winners of the ANZIAM medal each to submit a paper for publication in the journal. Dr. Y. M. Stokes was asked to look for unpublished work of the late Professor Tuck, who was awarded the medal in 1999, that might be suitable for posthumous publication, perhaps with some editing. This almost complete review article was found in a search of the excellent webpage he maintained and, with the help of Professor Tuck's wife, Mrs. Helen Tuck, the source files were located on his computer. The IMA/BAMC was approached and consented to its publication. After a search for an appropriate editor, an editorial committee of three was agreed upon, comprising Professor C. C. Mei, Professor J. N. Newman, and Dr. Y. M. Stokes, all former colleagues of Professor Tuck.

Only minor editing was required and the paper is almost exactly as written by Professor Tuck. The conclusions and acknowledgements sections, which in the original draft contained only the words "to be written", have been removed. We did not think it possible to write these sections as Professor Tuck would have done. Rather we decided to add this editorial note explaining the origin of the paper and the reason for the lack of the usual conclusions and acknowledgements. Dr. Stokes would here like to express her sincere thanks for the editorial assistance of Professors Mei and Newman, without which this paper would not have been published. 
Every effort has been made to secure permission to reproduce the photograph in Figure 2, but it has proved impossible to trace the copyright holder. If any omission is brought to our notice, we will be happy to include an appropriate acknowledgement on reprinting (in any subsequent edition).

We trust that readers will, like us, find the paper interesting and enjoyable, written, as it is, in the much-loved, exuberant presentation style of the late Professor Tuck.

\section{References}

[1] P. R. Garabedian, "Calculation of axially symmetric cavities and jets", Pacific J. Math. 6 (1956) 611-684; http://projecteuclid.org/euclid.pjm/1103043794.

[2] J. J. Kalker, "On elastic line contact”, J. Appl. Mech. 39 (1972) 1125-1132; doi:10.1115/1.3422841.

[3] T. von Kármán and N. B. Moore, "Resistance of slender bodies moving with supersonic velocities, with special reference to projectiles", Trans. ASME 54 (1932) 303-310.

[4] H. Lamb, Hydrodynamics, 6th edn (Cambridge University Press, Cambridge, 1932).

[5] M. J. Lighthill, "Supersonic flow past bodies of revolution", Aeronautical Research Council's Reports and Memoranda No. 2003 (1945).

[6] M. J. Lighthill, "A technique for rendering approximate solutions to physical problems uniformly valid", Phil. Mag. Ser. 740 (1949) 1179-1201; doi:10.1080/147864449008561410.

[7] H. Maruo, "Calculation of the wave resistance of ships, the draught of which is as small as the beam", J. Zosen Kiokai 112 (1962) 21-37; doi:10.2534/jjasnaoe1952.1962.112_21.

[8] A. May, "Water entry and the cavity-running behavior of missiles", SEAHAC Technical Report 75-2, NAVSEA Hydroballistics Advisory Committee, Silver Spring, Maryland, 1975.

[9] J.-M. Michel (ed.) Supercavitating flows, RTO Lecture Series 005, RTO-EN-010 (NATO Research and Technology Organisation, Neuilly-sur-Seine, 2002); http://www.rto.nato.int/Pubs/rdp.asp?RDP=RTO-EN-010.

[10] J. H. Michell, "The wave-resistance of a ship", Phil. Mag. Ser. 545 (1898) 106-123; doi:10.1080/14786449808621111.

[11] B. Molin, "On the piston and sloshing modes in moonpools", J. Fluid Mech. 430 (2001) 27-50; doi:10.1017/S0022112000002871.

[12] C. Panek and J. J. Kalker, "A solution for the narrow rectangular punch", J. Elasticity 7 (1977) 213-218; doi:10.1007/BF00041093.

[13] H. Reichardt, The laws of cavitation bubbles at axially symmetrical bodies in a flow, Volume 766 of Reports and Translations (Ministry of Aircraft Production, Moscow, 1946).

[14] Yu. N. Savchenko, "Experimental investigation of supercavitating motion of bodies", in: Supercavitating flows, RTO Lecture Series 005, RTO-EN-010 (ed. J.-M. Michel), (NATO Research and Technology Organisation, Neuilly-sur-Seine, 2002); 4-1-4-24; http://www.rto.nato.int/Pubs/rdp.asp?RDP=RTO-EN-010.

[15] E. O. Tuck, "The steady motion of a slender ship", Ph.D. Thesis, Cambridge University, 1963.

[16] E. O. Tuck, "Some methods for flows past blunt slender bodies", J. Fluid Mech. 18 (1964) 619-635; doi:10.1017/S0022112064000453.

[17] E. O. Tuck, "Toward the calculation and minimization of Stokes drag on bodies of arbitrary shape", 3rd Australasian Conference on Hydraulics and Fluid Mechanics (Institute of Engineers, Australia, 1970) 29-32.

[18] E. O. Tuck and C. C. Mei, "Contact of one or more slender bodies with an elastic half space", Internat. J. Solids Structures 19 (1983) 1-23; doi:10.1016/0020-7683(83)90034-3.

[19] E. O. Tuck and J. N. Newman, "Longitudinal waves in slender moonpools", in: 17th International Workshop on Water Waves and Floating Bodies (ed. R. Rainey), (Royal Institution of Naval Architects, London, 2002). 
[20] E. O. Tuck, D. C. Scullen and L. Lazauskas, "Wave patterns and minimum wave resistance for high-speed vessels", in: 24th Symposium on Naval Hydrodynamics (Office of Naval Research, Washington, DC, 2002).

[21] M. P. Tulin, "Steady two-dimensional cavity flows about slender bodies", Report No. 834, Navy Department, David W. Taylor Model Basin, 1953.

[22] M. P. Tulin, "On the shape and dimensions of three-dimensional cavities in supercavitating flows", Appl. Sci. Res. 58 (1998) 51-61; doi:10.1023/A:1000707013033.

[23] G. Vossers, "Some applications of the slender body theory in ship hydrodynamics", Ph.D. Thesis, Delft University, 1962.

[24] G. N. Ward, Linearised theory of steady high-speed flow (Cambridge University Press, Cambridge, 1955).

[25] J. V. Wehausen and E. V. Laitone, Surface waves, Volume IX of Encyclopedia of Physics (ed. S. Flügge), (Springer, Berlin, 1960) 446-778; http://coe.berkeley.edu/SurfaceWaves/. 